Guiding Principles for Water Quality and Aquatic Ecosystem Monitoring

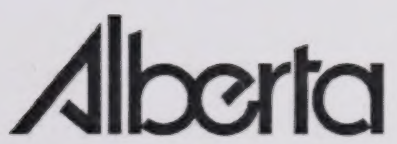

Environment 
Digitized by the Internet Archive in 2016

https://archive.org/details/guidingprinciple00watk 


\title{
Guiding Principles for Water Quality and Aquatic Ecosystem Monitoring
}

\author{
Prepared by:
}

Alberta Environment

July 2006 
ISBN: 0-7785-5083-4 (Printed Edition)

ISBN: 0-7785-5084-2 (On-line Edition)

Web Site: http://www3.gov.ab.ca/env/info/infocentre/publist.cfm

Any comments, questions or suggestions regarding the content of this document may be directed to:

Environmental Monitoring and Evaluation Branch

Environmental Assurance Division

Alberta Environment

12th Floor, Oxbridge Place

9820 - 106 Street

Edmonton, Alberta T5K 2J6

Fax: (780) 422-8606

Additional copies of this document may be obtained by contacting:

Information Centre

Alberta Environment

Main Floor, Oxbridge Place

9820 - 106 Street

Edmonton, Alberta T5K 2J6

Phone: (780) 427-2700

Fax: (780) 422-4086

Email: env.infocent@gov.ab.ca 
This report was prepared by a working group chaired by: Elise Watkins

Group members listed alphabetically

Anne-Marie Anderson

Theo Charette

Brian Jackson

Wendell Koning

Chris Teichreb

David Trew 


\section{TABLE OF CONTENTS}

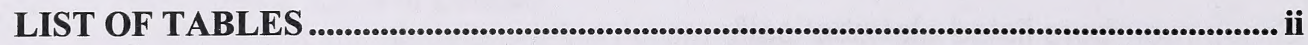

LIST OF FIGURES $\ldots . \ldots \ldots \ldots \ldots \ldots \ldots \ldots \ldots \ldots \ldots \ldots \ldots \ldots \ldots \ldots \ldots \ldots \ldots \ldots \ldots \ldots \ldots \ldots \ldots \ldots \ldots \ldots \ldots \ldots \ldots \ldots \ldots \ldots \ldots \ldots \ldots \ldots \ldots \ldots$ ii

VISION STATEMENT $\ldots \ldots \ldots \ldots \ldots \ldots \ldots \ldots \ldots \ldots \ldots \ldots \ldots \ldots \ldots \ldots \ldots \ldots \ldots \ldots \ldots \ldots \ldots \ldots \ldots \ldots \ldots \ldots \ldots \ldots \ldots \ldots \ldots \ldots \ldots \ldots \ldots 1$

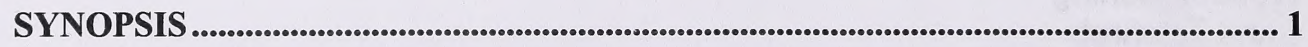

1. INTRODUCTION

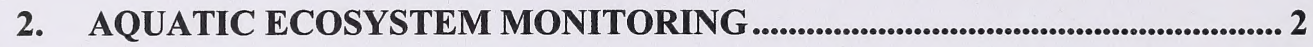

3. ASSURING HIGH QUALITY INFORMATION $\ldots \ldots \ldots \ldots \ldots \ldots \ldots \ldots \ldots \ldots \ldots \ldots \ldots \ldots \ldots \ldots . . . \ldots 6$

4. RISK CONSIDERATIONS $\ldots \ldots \ldots \ldots \ldots \ldots \ldots \ldots \ldots \ldots \ldots \ldots \ldots \ldots \ldots \ldots \ldots \ldots \ldots \ldots \ldots \ldots \ldots \ldots \ldots \ldots \ldots \ldots \ldots . \ldots \ldots$

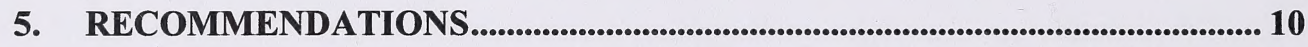




\section{LIST OF TABLES}

Table 1. Gradient of Complexity - What are the risks associated with compromised "water quality" data?

\section{LIST OF FIGURES}

Figure 1. Steps and Guiding Principles for Water Quality and Aquatic Ecosystem Monitoring 


\section{VISION STATEMENT}

\section{Water quality and aquatic ecosystems management in Alberta are based on high quality scientific information.}

\section{SYNOPSIS}

- The Alberta government's surface water quality monitoring program continues to evolve towards a more holistic, aquatic ecosystem monitoring approach.

- The program has defined steps for monitoring, which are based upon scientific protocols, operational principles and professional accountability.

- The program produces accurate, timely and reliable assessments of aquatic ecosystems to support a systematic approach to environmental management 


\section{INTRODUCTION}

The three goals of Water for Life: Alberta's Strategy for Sustainability (AENV 2003) are to ensure safe drinking water; healthy aquatic ecosystems; and reliable, quality water supplies for a sustainable economy. To fulfil these goals, the actions outlined in the strategy revolve around three key directions: knowledge and research; partnerships; and water conservation.

Access to reliable environmental information is therefore a key requirement to determining the success of Water for Life initiatives. Alberta Environment has been the primary source of water quality information in the past, but researchers, other monitoring agencies and volunteers are becoming more involved with generating this information.

AENV is expected to provide oversight to ensure the availability of reliable information and, ultimately, is accountable to meet the goals set out in Water for Life (Alberta Water Council, 2005). With the evolution of environmental governance and accountability concepts in Alberta, there is a need to clarify fundamental requirements of monitoring programs for all potential participants.

This document provides a short summary of the scientific steps and processes involved in aquatic ecosystem monitoring. (The term "monitoring" is used here generically to represent various types and scales of data collection activities, including regional surveys, long-term fixed-site monitoring, research support, etc.) It spells out guiding principles and the accountability considerations necessary to ensure that high quality, scientific, reliable information is available to make informed decisions regarding aquatic ecosystem and watershed management.

\section{AQUATIC ECOSYSTEM MONITORING}

Aquatic ecosystem monitoring activities encompass a range of water quality, sediment quality and aquatic biota assessments. Among other considerations, the design assumes a current knowledge of sources and types of contaminants and an understanding of the interactions between climate, hydrology and watershed processes.

Monitoring information is used to support the broad goals of Water for Life by providing a knowledge base to support a diverse range of watershed management activities, including: predictive modelling for wastewater regulation; water allocation decisions and infrastructure operations; development of ambient guidelines and in-stream targets to support watershed planning, environmental performance measurement and state of the environment (SOE) reporting; negotiation of trans-boundary water management agreements; and research into a wide variety of issues including aquatic ecosystem contamination, use impairment and restoration.

An overarching principle for the monitoring of Alberta's aquatic ecosystems is that high quality scientific information will be available to ensure the success of Water for Life, a strategy for which the Minister of the Environment is ultimately accountable. 
Monitoring consists of a sequence of closely linked and inter-dependent steps conducted in the context of established operational principles (Figure 1):

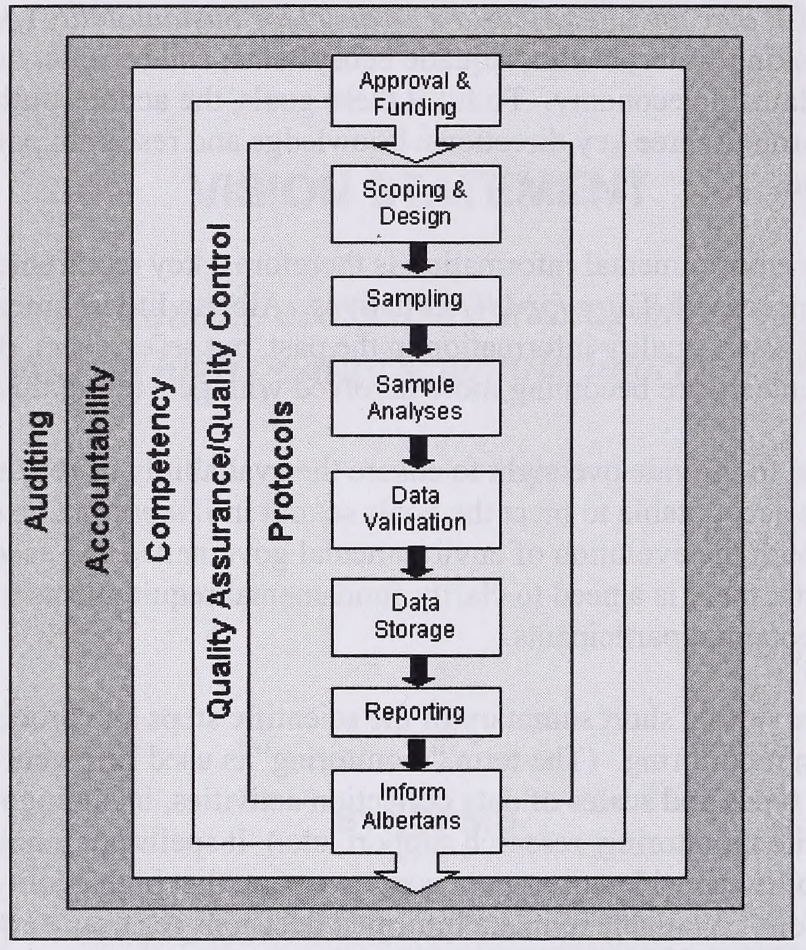

Figure 1. Steps and Guiding Principles for Water Quality and Aquatic Ecosystem Monitoring

1. Scoping and Design is based
on clear scientific understanding
of:
- Issues;
Relevant background
information;
Study objectives;
$\circ$ Desired outcomes;
$\circ$ Appropriate methods;
$\circ$ The dynamics and
$\quad$ characteristics of water
systems.

The scoping and design step is the foundation of all monitoring studies. During the scoping phase study objectives are defined based on a clear understanding and knowledge of issues, relevant scientific information and the characteristic of the aquatic environments in question. The study design specifies: when, where and how to sample; who should analyze the samples and what methods to employ. It outlines quality assurance/quality control (QA/QC) steps and how the data will be managed to support the final interpretation. Minimizing sampling costs while optimizing the value of data collected is an inherent design step.

The study design needs to be shared among potential partners and stakeholders to ensure a common understanding of goals, requirements and limitations of the study. Once a project is approved, resources (funds and manpower) need to be allocated so the project is implemented according to plan and within the appropriate time window. 
Project managers have the responsibility to ensure the project is executed according to the plan. To achieve this they must: stay in close communication with the field staff in charge of implementing the project; communicate with analytical laboratories; participate in data validation; ensure that data storage is proceeding and that data are analyzed; and communicate results to partners and stakeholders.

\section{Sample Collection requires} training, expertise, skills and adherence to:

- Project design;

- Defined methodologies (standard operating protocols);

- Data management standards;

- Health and Safety considerations.
The goal of this step is to collect samples or information that meet design specifications without compromising the safety of those involved. The field project manager is in ongoing communication with the project manager and coordinates the sampling program. This involves:

- Maintaining equipment, preparing sampling gear, and ensuring sample containers are properly labelled and coded and analytical labs are prepared to receive and process the new samples;

- Collecting samples according to the study design and adhering to QA/QC specifications;

- Transporting samples to the appropriate labs with accurate documentation (analytical request forms), and following up with lab managers as necessary;

- Ensuring that field notes are completed and copied to the project manager in a timely fashion;

- Entering field information into electronic records;

- Validating analytical data sheets as they come back from the project manager.

\section{Sample Analysis:}

- Provides data that meet the needs of the study design and that will stand up to current and future scrutiny;

- Follows established, documented protocols;

- Relies on sufficient $Q A / Q C$ to ensure data quality;

- Reported in a timely fashion.

\section{Chemical, biological and physical}

analyses must be performed by competent laboratories and individuals. Results must meet scientific criteria. Laboratories involved in processing environmental samples must follow acceptable, documented procedures. Laboratories must be experienced in conducting the specified analyses and have appropriate accreditation (e.g., CAEL) or be recognized as experts for specialized analyses.

The laboratory manager must be in contact with the project manager and discuss any departures from agreed-upon methods and procedures. Typical functions associated with sample analyses include: 
- Reception and cataloguing of new samples followed by appropriate storage until analysis;

- Analyses of samples within appropriate time limits and according to the analytical request sheets;

- Communicating results to the project manager in a format that is mutually agreeable;

- Sending invoices to project manager;

- Resolution of QA/QC issues such as data transfer errors and repeat analyses;

\section{Data validation ensures the} reliability of the data before they become publicly available. This requires:

- Validation of field and laboratory information;

- Validation of database entries;

- Potential involvement of project, field and laboratory managers as well as the data manager.
The data validation step requires those involved in the collection of samples and generation of data (project manager, field staff, laboratories, and data managers) to double check all entries. The data validation procedure is required at all steps of data generation, from field notes to database entries. A final check and signoff by the project manager must occur before the data are released electronically.

Routine checks and actions involve:

- Completeness of the data (sites, dates, variables);

- Ensuring the appropriateness of field and laboratory methods, and the adequacy of coding and labelling;

- Scrutinizing the results and QA/QC information and, where necessary, requesting repeat analyses from the laboratory;

- Ensuring that all information has been included on the water data system (WDS) and appropriately characterized (i.e., QA/QC sample, spiking information, true sample, method and site codes);

- Providing the $\mathrm{QA} / \mathrm{QC}$ information to the laboratory promptly. This is an important step in data validation. It allows the laboratory to keep track of ongoing analytical performance and make any necessary adjustments in a timely fashion. 


\section{Data Storage ensures that:}

- Information and data are stored reliably over the longterm;

- Information and data are accessible to all interested parties.
Appropriate data storage is a key step in the monitoring process as it ensures data are securely stored over the long-term and data are readily available to all parties.

- It ensures that a minimum set of criteria needed for storage on the database have been met and that the originator of the data can be clearly identified.

- Well-trained and knowledgeable information technology staff must conduct data storage and database management.

- Well-maintained, centralised databases are preferable to a proliferation of independent databases in order to ensure the integrity of the data and consistent validation procedures.

\section{Reporting involves the} accurate, reliable, unbiased and scientifically defensible conversion of data into information in a timely manner, by competent parties.
Data must be converted to accurate, reliable and scientifically defensible information and reported in a timely manner:

- Competent and experienced individuals are required for the data analysis and reporting step;

- Data analysis techniques and criteria outlined in the study design ensure results can be reported in an unbiased manner;

- The objectives of the study need to be addressed and the limitation of the data need to be recognized;

- Reporting can take the form of written documents or verbal communication and needs to take into consideration the level of language of the intended audience;

- Scientific peer reviews will further ensure the accuracy and reliability of the reported information.

- The release process must respect the public need for timely, unbiased, and accurate information.

\section{ASSURING HIGH QUALITY INFORMATION}

To ensure the overall and ongoing success of monitoring programs there is a need to develop concrete tools, procedures and philosophical guidance that help assure the validity and quality of information. 


\section{Protocols Written documents}

that provide step-by-step

descriptions needed to complete specific actions.
Protocols are written documents that describe accepted approaches necessary to fulfil certain tasks. The study design identifies and integrates applicable protocols in a prescriptive manner. Protocols are needed at each step of monitoring and documents need to be made available,

understood and implemented by all those involved in monitoring activities. Examples of protocols are:

- Standard Operating Procedures (SOPs) for the collection of samples,

- SOPs for the analysis of specific water or sediment quality variables, or the processing of biological samples

- SOPs for field and laboratory data management;

- Acceptance or rejection of laboratory results based on QA/QC information;

- Occupational health and safety documents

- Hazardous assessment documents

Although many protocols are available, there is an ongoing need to develop protocols as new equipment and methods become available for environmental monitoring.

\section{Quality Assurance Plan $A$ defined plan to ensure the precision, accuracy, completeness and representativeness of produced data.}

A Quality Assurance Program (QAP) for monitoring programs is a management tool that helps guarantee data are of sufficient quality to withstand scientific (and legal) challenges relative to the use for which the data are obtained. The needs for quality assurance/quality control $(\mathrm{QA} / \mathrm{QC})$ measures at all levels of

monitoring programs are well recognized. Typical steps include adherence to protocols, inclusion of QA/QC samples in the field and laboratory, and peer or expert reviews of study designs and reports. When data originate from multiple stakeholders, the existence of a centralized QAP provides a coordinated and standardized approach by: formalizing $\mathrm{QA} / \mathrm{QC}$ measures; clarifying accountability issues; and specifying data quality objectives.

\section{Competency The training, skill and experience necessary to perform specific tasks and to understand their importance.}

Competency requirements will vary depending on the nature of the study and the monitoring steps involved. Training requirements may be relatively simple when collecting samples for educational studies of an elementary nature. However, skill and training level will be higher

for studies that need to generate scientifically defensible data, particularly if the use and maintenance of sophisticated equipment is required. In these situations, professional accreditation or certification is required. All staff and partners involved in monitoring programs must comply with occupational health and safety guidelines and have completed the required safety courses. 


\section{Accountability The definition and acceptance of professional responsibility for the proper execution of one or more steps in the monitoring process.}

Each manager (i.e., project, field, laboratory, data) is accountable for the successful completion of individual monitoring steps. Accountability issues apply equally to: field, laboratory and data management staff; professionals who design projects, interpret and communicate information; and agencies involved

in the funding of projects and/or release of data and reports. The definition of accountability ensures the successful completion of projects, and provides an assurance that environmental decisions are based on sound monitoring information. Accountability issues need to be clarified at the onset of each project.

\section{Auditing A periodic, methodical and independent examination of monitoring programs.}

There is a need to instate an independent auditing system to ensure, through periodic checks, that protocols and accountability chains are adhered to and the quality of information used to make environmental decisions is

sufficient, pertinent and of high quality. Auditing provides a vehicle to report on performance, and identifies the need for ongoing training and improvement.

\section{RISK CONSIDERATIONS}

Even with full adherence to study design and protocols there is a possibility errors, omissions, or mishaps will occur that may occasionally compromise the integrity of the information. Errors may only be discovered during the iterative, peer review activities of ongoing scientific studies. The greatest concern is that the lack of integrity of the information may never be recognized and that erroneous conclusions will be drawn, resulting in faulty watershed management decisions.

The implications of inappropriate management decisions, based on compromised information, may vary depending on the nature and objectives of the study. As stated above, a simple education project, geared for the elementary or junior high school level, may have relatively little consequence for society if full scientific protocols are not followed and results are unreliable. At the other end of the spectrum, a complex study of environmental contamination, with direct relevance for human health evaluations, may have considerable risk for society if the information produced is compromised. A simple depiction of complexity and risk considerations is illustrated in Table 1. Measures to mitigate information risks need to be considered carefully at the onset of environmental sampling programs. 


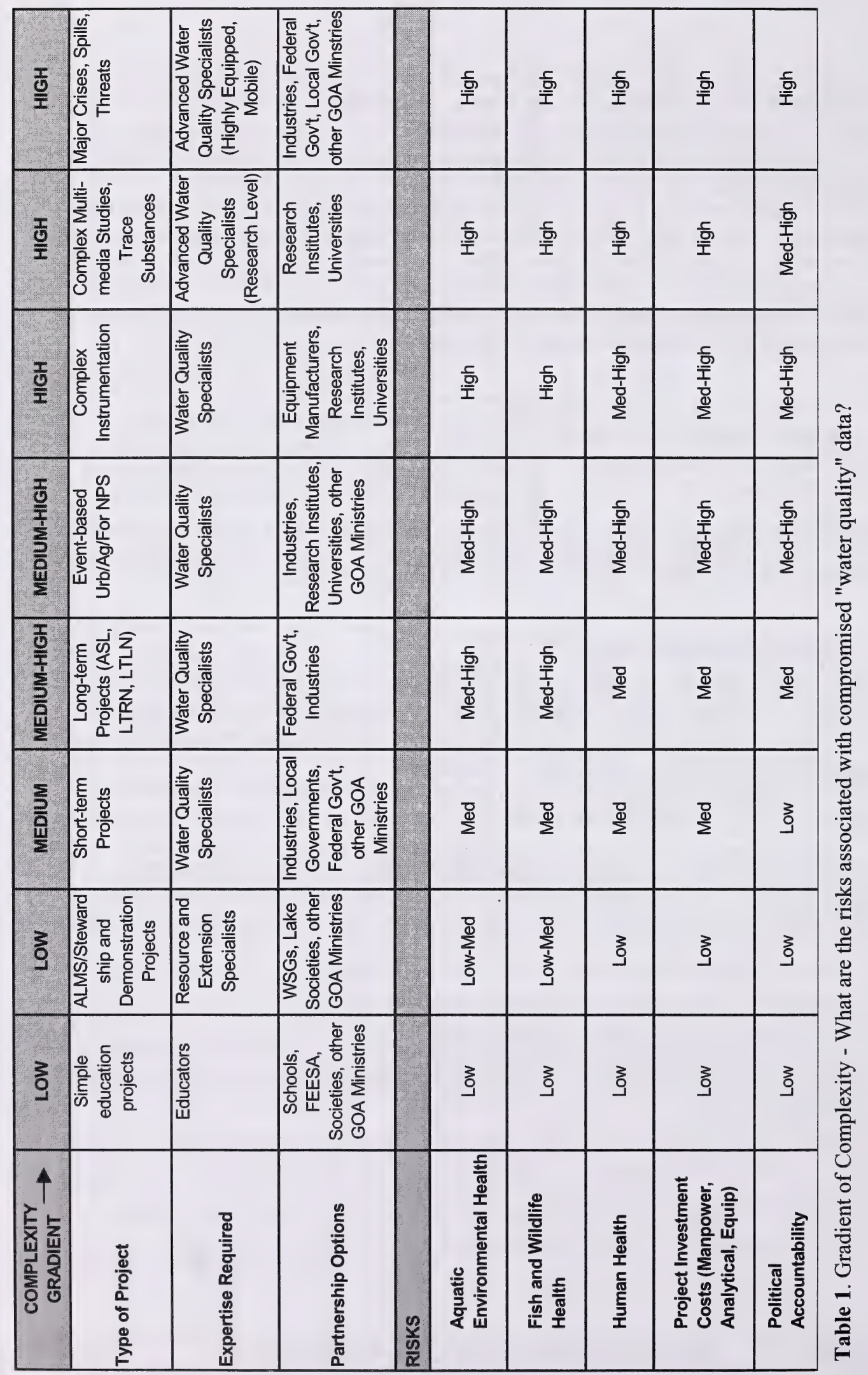




\section{RECOMMENDATIONS}

Alberta Environment has conducted surface water quality monitoring programs for many years, both independently and in cooperation with a relatively small number of partners. As the number of partners involved in generating information and data on aquatic ecosystems increases in the future it will be important that an adequate support framework is in place to ensure that the information generated is of appropriate reliability and quality.

This support framework needs to include:

- A comprehensive set of protocols for all components of monitoring;

- A description of competency requirements for various types of studies and aspects of monitoring;

- Appropriate training of partners in the relevant steps of monitoring;

- A centralized quality assurance plan (to be formalized by the agency which has the ultimate accountability of the information, i.e., Alberta Environment);

- An accountability system so that all partners clearly understand their roles and responsibilities;

- An auditing system. 


LIBRARY AND ARCHIVES CANADA
Bibliothèque et Archives Canada

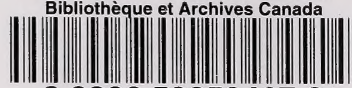

33286538594678 